\title{
The analitical study of a biphase cadmium selenide structural model
}

\author{
V.S. Grinevich, V.M. Vorobel, V.A. Smyntyna, L.N. Filevskaya \\ I.I. Mechnikov Odessa National University, 2, Dvorjanskaya str., 65026 Odessa, Ukraine \\ Phone/fax: 38-048-731-74-03, \\ E-mail: grinvict@mail.ru
}

\begin{abstract}
The elastic interactions of two CdSe structural modifications (cubic and hexagonal) were studied for the comprehensive analysis of polycrystalline cadmium selenide parameter degradation. The system studied was modeled as a hexagonal matrix with a spherical inclusion of the cubic modification where elastic strains were calculated at the matrix-inclusion boundary. The fulfilled analysis permitted, besides the main problem solution, to obtain the elastic constants $\mu$ and $K$ for two modifications of CdSe, which essentially supplies the known material characteristics. The top value of the pressure, $P_{0}$ normal component, achieves $10^{9} \mathrm{~Pa}$, which excesses over the known values of $P_{0}$. The results achieved may be useful for the further investigations of the degradation transformations in a polycrystalline biphase systems.
\end{abstract}

Keywords: structural transformations, elastic parameters, cadmium selenide.

Manuscript received 10.02.05; accepted for publication 18.05.05.

The degradation problems are of principle at the construction of electronic devices [1]. In their own turn, the degradation processes in biphase materials are to a considerable extent defined by differences in structural properties of the phases that come into contact with each other. This leads to appearance of considerable mechanical strains, which influence the chemical bond states at the interphase boundary.

In the work [2], semiconductor polycrystalline cadmium selenide layers are reported to be investigated. As a result, the model of structural transformations in the system: hexagonal $\mathrm{CdSe}(\alpha-\mathrm{CdSe})-$ as a matrix and cubic $\mathrm{CdSe}(\beta$-CdSe) - as a spherical inclusion was approbated [3]. It was established that in the considered system, the elastic strains exist at the matrix - inclusion boundary. In this work, developing the previously reported ideas, made is an attempt of a detailed analysis of the suggested model by means of the elasticity theory methods.

We shall consider an infinite elastic medium $(\alpha$-CdSe) with a spherical type elastic inclusion of $\beta$-CdSe of $R_{0}$ radius in a spherical coordinate system (figure). For the simplicity, we shall consider that the deformation and strains depend upon the variable $r$ only.

At the condition of mass forces (gravitational, inertial) absence in the spherical coordinate system and taking into account the central symmetry, the equilibrium equation will have be [4], when deformations and strains depend only on the inclusion radius $r$ : $\frac{d}{d r}\left[\frac{1}{r^{2}} \cdot \frac{d\left(r^{2} u\right)}{d r}\right]=0$,

where $u(r)$ - the body particles displacement along the $r$-axis. The general solution of the equation (1) and of radial strain may be written down as follows [4]:

$u=a r+\frac{b}{r^{2}}$

$\sigma_{r r}=3 K a-4 \mu \frac{b}{r^{2}}$

where $a, b$ are constants, that will be determined later; $K=\frac{E}{3(1-2 \sigma)}-$ modulus of dilatation, $\mu=\frac{E}{2(1+\sigma)}-$ shear modulus, $E$ - Young's modulus, $\sigma-$ Poisson's ratio.

Due to the boundary conditions, in the origin of coordinates and in infinity, the general solution for the matrix and for the inclusion is simplified:

$u_{i}=a_{i} \cdot r$

$\sigma_{r r}^{i}=3 K_{i} \cdot a_{i}$

$u_{m}=\frac{b_{m}}{r^{2}}$ 


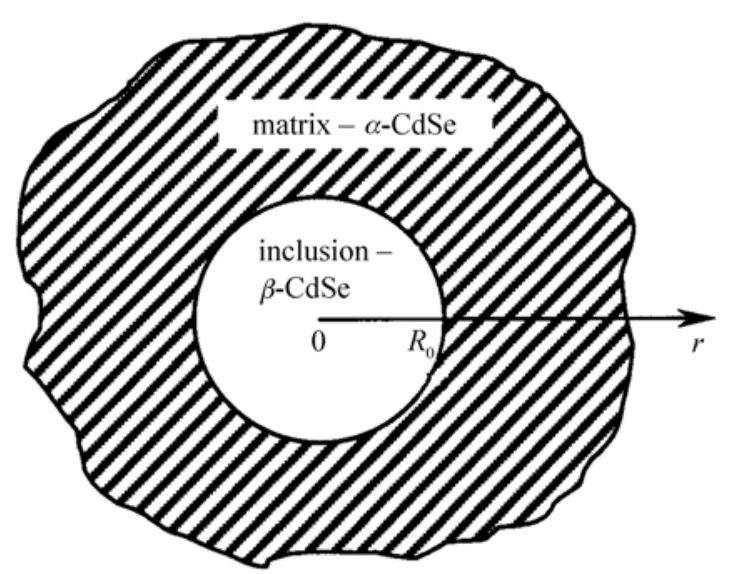

Schematic presentation of the modeled object.

$\sigma_{r r}^{m}=4 \mu_{m} \cdot \frac{b_{m}}{r^{3}}$

where $i$ and $m$ indexes given to the values which correspond to the inclusion and to the matrix, respectively.

Let us consider the following boundary conditions:

$\left.u_{m}\right|_{r=R_{0}}-\left.u_{m}\right|_{r=R_{0}}=u_{0}$,

$\left.\sigma_{r r}^{m}\right|_{r=R_{0}}=\left.\sigma_{r r}^{i}\right|_{r=R_{0}}$,

where $u_{0}$ - the initial deformation of the inclusion.

After the corresponding substitution of $u_{m}$ from (6) and of $u_{i}$ from (4) into (8), $\sigma_{r r}^{m}$ from (7) and $\sigma_{r r}^{i}$ from (5) into (9), we shall obtain the system of following equations:

$a_{i} R_{0}^{3}-b_{m}=-u_{0} R_{0}^{2}$,

$3 K_{i} R_{0}^{3} \cdot a_{i}+4 \mu_{m} b_{m}=0$.

The solution of the system gives:

$b=\frac{u_{0} 3 K_{i} R_{0}^{2}}{4 \mu_{m}+3 K_{i}}, a=\frac{u_{0} 4 \mu_{m}}{R_{0}\left(4 \mu_{m}+3 K_{i}\right)}$.

Taking into consideration (11), the strain at the matrix inclusion boundary will be:

$\left.\sigma_{r r}^{i}\right|_{r=R_{0}}=P_{0}=\frac{u_{0} 12 \mu_{m} K_{i}}{R_{0}\left(4 \mu_{m}+3 K_{i}\right)}$.

It we consider $u_{0}=\Delta R$, then the inclusion volume relative variation is:

$$
\begin{aligned}
& \frac{\Delta V}{V}=\frac{\left(R_{0}+\Delta R\right)^{3}}{R_{0}^{3}}= \\
& =3 \frac{\Delta R}{R_{0}}+3\left(\frac{\Delta R}{R_{0}}\right)^{2}+\left(\frac{\Delta R}{R_{0}}\right)^{3} .
\end{aligned}
$$

Considering $\frac{\Delta R}{R}$ as a little value, we may accept that $\frac{u_{0}}{R_{0}} \approx \frac{\Delta V}{3 V}$ for the quantative evaluation, then

$$
P_{0}=\frac{4 K_{i} \mu_{m}}{\left(3 K_{i}+4 \mu_{m}\right)} \cdot \frac{\Delta V}{V} .
$$

Knowing the elastic compliance $s_{i j}$ and elastic rigidity $c_{i j}$ constants for the hexagonal single crystals $\alpha$-CdSe $\left(c_{11}=7.49, \quad c_{12}=4.609, \quad c_{13}=3.926, c_{33}=8.451, c_{44}=\right.$ $\left.=1.315, c_{66}=1.441\right) \cdot 10^{10} \mathrm{~Pa},\left(s_{11}=3.327, s_{12}=-1.144\right.$, $\left.s_{13}=-0.5498, \quad s_{33}=1.694, \quad s_{44}=7.605, \quad s_{66}=6.939\right) \times$ $\times 10^{-11} 1 / \mathrm{Pa}$, and also for cubic $\beta$-CdSe $\left(c_{11}=7.4\right.$, $\left.c_{12}=4.52, c_{44}=1.32\right) \cdot 10^{10} \mathrm{~Pa},\left(s_{11}=2.517, s_{12}=-0.9546\right.$, $\left.s_{44}=7.57\right) \cdot 10^{-11} 1 / \mathrm{Pa}[5]$, we may calculate the mean values of modulus of dilatation $K_{i}$ and shear modulus $\mu_{m}$. The calculation will be realized by the Foyght and Royss methods [6]. As a results for $\alpha$-CdSe will have according to Foyght:

$K_{m F}=\frac{1}{9}\left(2 c_{11}+c_{33}+4 c_{13}+2 c_{12}\right)=5.373 \cdot 10^{10} \mathrm{~Pa}$,

$\mu_{m F}=\frac{1}{30}\left(7 c_{11}+2 c_{33}-5 c_{12}-4 c_{13}+12 c_{44}\right)=$

$=1.545 \cdot 10^{10} \mathrm{~Pa}$,

according to Royss:

$\frac{1}{K_{m R}}=\left(2 s_{11}+s_{33}+s_{12}+4 s_{13}\right)$,

$K_{m R}=5.374 \cdot 10^{10} \mathrm{~Pa}$,

$\frac{1}{\mu_{m R}}=\frac{2}{15}\left(7 s_{11}+2 s_{33}+3 s_{44}-5 s_{12} 54 s_{13}\right)$,

$\mu_{m R}=1.293 \cdot 10^{10} \mathrm{~Pa}$.

For $\beta$-CdSe according to Foyght:

$K_{i F}=\frac{1}{3}\left(c_{11}+2 c_{12}\right)=5.48 \cdot 10^{10} \mathrm{~Pa}$,

$\mu_{i F}=\frac{1}{5}\left(c_{11}-c_{12}+3 c_{44}\right)=1.368 \cdot 10^{10} \mathrm{~Pa}$,

according to Royss:

$\frac{1}{K_{m R}}=\left(2 s_{11}+s_{33}+s_{12}+4 s_{13}\right)$,

$K_{m R}=5.374 \cdot 10^{10} \mathrm{~Pa}$,

$\frac{1}{K_{i R}}=3\left(s_{11}+2 s_{12}\right)$,

$\frac{1}{\mu_{i R}}=\frac{1}{5} 4\left[\left(s_{11}-s_{12}\right)+3 s_{44}\right]$,

$K_{i R}=5.48 \cdot 10^{18} \mathrm{~Pa} ; \mu_{i R}=1.366 \cdot 10^{10} \mathrm{~Pa}$.

The $K$ and $\mu$ values are given in Table 1 . 
Table 1

\begin{tabular}{|l|l|l|}
\hline & $K, \mathrm{~Pa}$ & $\mu, \mathrm{Pa}$ \\
\hline$\alpha$-CdSe & $(5.373-5.374) \cdot 10^{10}$ & $(1.293-1.545) \cdot 10^{10}$ \\
\hline$\beta$-CdSe & $5.48 \cdot 10^{10}$ & $(1.366-1.368) \cdot 10^{10}$ \\
\hline
\end{tabular}

The data given in Table 1 gives the possibility to obtain the values of $E=\frac{9 K \mu}{3 K+\mu}$ and $\sigma=\frac{3 K-2 \mu}{2(3 K+\mu)}$ for hexagonal and cubic modifications, respectively (Table 2).

Table 2

\begin{tabular}{|c|c|c|c|}
\hline & & $E, \mathrm{~Pa}$ & $\sigma$ \\
\hline \multirow{2}{*}{$\alpha$-CdSe } & $\begin{array}{l}\text { according to } \\
\text { Foyght }\end{array}$ & $4.231 \cdot 10^{10}$ & 0.369 \\
\hline & $\begin{array}{l}\text { according to } \\
\text { Royss }\end{array}$ & $3.59 \cdot 10^{10}$ & 0.389 \\
\hline \multirow{2}{*}{$\beta$-CdSe } & $\begin{array}{l}\text { according to } \\
\text { Foyght }\end{array}$ & $3.789 \cdot 10^{10}$ & 0.385 \\
\hline & $\begin{array}{l}\text { according to } \\
\text { Royss }\end{array}$ & $3.782 \cdot 10^{10}$ & 0.385 \\
\hline
\end{tabular}

The experimental value of $E$ for $\alpha$-CdSe: $E=5 \cdot 10^{10} \mathrm{~Pa}$ witnesses a reasonable correspondence with the calculated results, see Table 2 .

Let us estimate the possible elastic strains at the double-phase boundary, according to the formula (14) and to Table 1: $P_{0} \approx-1.4 \cdot 10^{10} \frac{\Delta V}{V} \mathrm{~Pa}$. For example, if $\Delta V=0.1 \mathrm{~V}$ then $P_{0}=-1.4 \cdot 10^{9} \mathrm{~Pa}$.

\section{Conclusions}

Our analysis based on the elasticity theory methods and applied to the biphase polycrystalline cadmium selenide layer showed the considerable strains $P_{0}$ present at the double-phase boundary. It is essential, that these strains may be initiated by different factors: the cubic phase inclusion growth, hexagonal matrix compression, shift caused by different temperature coefficients of the substrate, of the layer and each of the phases. It was ascertained [7] that structural transformation in CdSe takes place at $P=3 \cdot 10^{9} \mathrm{~Pa}$, which has reasonable correlation with $P_{0}$ values obtained in the present work. The obtained value of $P_{0}$ is enough for the mechanical destruction of the phase boundary with the correspondent chemical bonds break between these two modifications. The results obtained have the satisfactory agreement with the idea that the bond break takes place most probably at the boundary, because the regular bonds in each modification separately are more tight than those in the contact regions of the modifications.

The reported suppositions are confirmed by the experimental structure investigations [8], where the interplanes distances in heterophase structures differ from their standard values. The considerable error between the lattice constants $a$ for $\alpha$-CdSe and $\beta$-CdSe [5], where $\Delta a / a \approx 0.4$ is a factor of a considerable force appearance, which may stimulate structure transformations [9].

Hence, the analytical study of the structural model suggested may be an effective basis to investigate degradation processes in the structure and electrophysical parameters of layer.

\section{References}

1. B.J. Fitzpatrick and Tuoh-Bin Ng, Degradation Problems in II-VI Compounds // $11^{\text {th }}$ Intern. Conf. on II-VI Compounds. Niagara Falls, New York, USA, September 22-26, 2003. Book of Abstracts, No 1.1 (2003).

2. V.S. Grinevich, Thesis of candidate of science, 204 p. (1990).

3. V.S. Grinevich, V.A. Smyntyna, and L.N. Filevskaya, Correlation between electro-physical characteristics and elastic properties of cadmium selenide films // Phys. stat. sol.(c), N4, p. 690-693 (2004).

4. L.D. Landau, E.M. Lifshitz, Theoretical physics. V.7. The theory of elasticity. Moscow, Nauka (1987) (in Russian).

5. Physical and chemical properties of semiconductors, Handbook. Moscow, Nauka (1979) (in Russian).

6. V.A. Kuchin, V.L. Ulyanov, Elastic and inelastic properties of crystals. Moscow, Energoatomizdat (1986) (in Russian).

7. Physics and Chemistry of the $\mathrm{A}^{\mathrm{II}} \mathrm{B}^{\mathrm{VI}}$ compounds, Ed. by S.A. Medvedev, Moscow, Mir (1970) (in Russian).

8. V.S. Grinevich, V.E. Polishchuk, V.V. Serdyuk, V.A. Smyntyna // Izvestiya AN USSR Neorg. Materials 8, p.1262-1266 (1982).

9. H. Mariette, Self-assembled quantum dot formation induced by surface energy change of a strained two-dimensional layer // $11^{\text {th }}$ Intern. Conf. on II-VI Compounds. Niagara Falls, New York, USA, September 22-26, 2003. Book of Abstracts, No 1.1 (2003). 